\title{
Impact of immunonutrition on radical cystectomy immunoresponse and outcomes; opportunity for peri-operative optimization
}

\author{
Marc A. Bjurlin ${ }^{1}$, Angela B. Smith ${ }^{2,3}$, William C. Huang ${ }^{1}$ \\ ${ }^{1}$ Division of Urologic Oncology, Department of Urology, NYU Langone Health, New York, NY, USA; ${ }^{2}$ Department of Urology, University of \\ North Carolina at Chapel Hill, Chapel Hill, North Carolina, USA; ${ }^{3}$ Multidisciplinary Genitourinary Oncology, Lineberger Comprehensive Cancer \\ Center, Chapel Hill, North Carolina, USA \\ Correspondence to: Marc A. Bjurlin, DO, MSc, FACOS. Division of Urologic Oncology, Department of Urology, NYU Langone Health, NYU \\ Langone Hospital-Brooklyn, 150 55th Street, Brooklyn, NY 11220, USA. Email: Marc.Bjurlin@nyumc.org. \\ Provenance: This is an invited Editorial commissioned by Section Editor Xiao Li (Department of Urology, Jiangsu Cancer Hospital \& Jiangsu \\ Institute of Cancer Research \& Nanjing Medical University Affiliated Cancer Hospital, Nanjing, China). \\ Comment on: Hamilton-Reeves JM, Stanley A, Bechtel MD, et al. Perioperative Immunonutrition Modulates Inflammatory Response after Radical \\ Cystectomy: Results of a Pilot Randomized Controlled Clinical Trial. J Urol 2018;200:292-301.
}

Submitted Jul 30, 2018. Accepted for publication Aug 07, 2018.

doi: 10.21037/tau.2018.08.09

View this article at: http://dx.doi.org/10.21037/tau.2018.08.09

Radical cystectomy with urinary diversion is the established 'gold standard' surgical treatment of muscle invasive bladder cancer. This procedure has one of the highest rates of morbidity, with adverse events occurring in up to twothirds of patients, regardless of surgical approach (open $v s$. robotic) (1). The significant peri-operative morbidity of radical cystectomy is driven by the complexity of the extirpative and reconstructive procedure, oncological risk factors, and peri-operative complications. A major preoperative reversible risk factor in surgery is malnutrition; up to $55 \%$ of radical cystectomy are at risk of pre-operative malnutrition (2). Poor nutritional status has been shown to be a risk factor for postoperative infections, poor wound healing, and complications (3-5). The immunological repercussions of malnourishment are significant; however, it appears some of these repercussions can be attenuated with diet and nutritional therapy in the peri-operative setting (6). Nutritional supplementation has now frequently been incorporated into the management of malnourished surgical patients.

The modulation of the immune system by supplemental nutrients is designated immunonutrition. The aim of immunonutrition is to supply nutrients to modify inflammatory or immune responses including arginine, glutamine, various amino acids, omega-3 polyunsaturated long-chain fatty acid, nucleotides and antioxidants. These substrates may play a role in the up-regulation of host immune responses, regulate inflammation, and influence protein synthesis. Arginine deficiency during immune deficiency has been shown to impact T-cell receptor complexes. Modulation of the immune system by supplemental nutrients may halt argininase-1, and increase levels of arginine to prevent immune deficiency, and potentially decrease postoperative complications (7). A period of immunosuppression following radical cystectomy may increase the risk of both morbidity and mortality. Recently, immunonutrition has been investigated as a means to improve the clinical course of bladder cancer patients and their response to surgical stress. The goal of early immunonutrition is to decrease the inflammatory response as opposed to enhancing it, to attenuate hyperinflammation, and prevent the compensatory immunosuppression with the goal of reducing complications.

Recently, Hamilton-Reeves et al. reported a pilot randomized controlled clinical trial comparing consumption of a specialized immunonutrition (SIM: a nutritional supplement of L-arginine, omega-3 fatty acids, and dietary nucleotides) to standard oral nutrition supplement (ONS: Boost Plus, consisting of $15 \%$ protein, $50 \%$ carbohydrate, and $35 \%$ fat along with 26 vitamins and minerals) for 5 days 
pre and post cystectomy on the acute inflammatory response and arginine level in bladder cancer patients who underwent radical cystectomy (8). A total of 29 men consumed SIM $(n=14)$ or ONS ( $n=15)$ ( 3 cartons per day) for 5 days before and after radical cystectomy for bladder cancer in a prospective, randomized protocol. T-helper (Th)1-Th2 balance, plasma interleukin (IL)-6, and plasma amino acids were measured at the start of the study, intraoperatively, and then postoperatively on days 2,14 , and 30 .

The authors reported several findings; using a validated instrument (PG-SGA) to assess malnutrition, the investigators noted over $25 \%$ of patients were moderately or severely malnourished, even after excluding patients with a body mass index $(\mathrm{BMI})<18.5$. Furthermore, this resulted in a $20.5 \%$ difference between PG-SGA and billing data, highlighting the inadequacies of nutritional support for patients undergoing radical cystectomy. In the SIM group, the study investigators observed a $54.3 \%$ average increase in Th1-Th2 balance from the start of the study to intra-operative day while Th1-Th2 balance decreased approximately $5 \%$ in the ONS group. This observed change in Th1-Th2 balance between the two cohorts was noted be significantly different $(\mathrm{P}<0.027)$. Plasma IL-6 was $42.8 \%$ lower following SIM compared to ONS on postoperative day $2(\mathrm{P}=0.020)$. SIM maintained plasma arginine from baseline to post-operative day 2, yet ONS showed $26.3 \%$ reduction from the study start to post-operative day $2(\mathrm{P}=0.0003)$. These results suggest that consuming SIM may counteract disrupted T-helper balance, decreases inflammatory response, and ultimately prevents arginine depletion from radical cystectomy.

In the same cohort, the authors have previously shown that the immune response to surgery and infection rates differ between patients receiving SIM versus standard ONS in the radical cystectomy perioperative period (9). Although the authors have found a substantial impact of SIM on study outcomes, controversy remains regarding the effect of peri-operative immunonutrition. Lyon et al. found immunonutrition supplementation was not associated with lower postoperative infectious complications in radical cystectomy patients (10). Maffezzini et al. found that immunonutrition had no influence on the recovery of biochemical markers of protein depletion when followed to post-surgical day 5 (11). In contrast, Bertrand et al. demonstrated immunonutrition in patients undergoing cystectomy for bladder cancer was associated with a decrease in postoperative complications including infections of the urinary tract, and paralytic ileus (6). The reasons for the contradictory results with immunonutrition in radical cystectomy patients require further elucidation. It remains unknown whether these differences are related to the heterogeneous nature of the study cohorts or to the composition of supplemental nutrients within the immunonutrition. Despite these apparent benefits of immunonutrition in these studies, no study has yet identified a significant effect of immunonutrition on mortality within surgical patients.

As Hamilton-Reeves and co-authors highlight, their study was limited by the small sample size (8). Additionally, participation at post-operative day 14 was poor, with limited collection of data at the follow-up points. Several additional questions remain regarding the implementation of immunonutrition in the radical cystectomy care pathway. It appears clear that supplemental nutrients are beneficial in the malnourished patient who undergoes high-risk surgery; even if patients are not malnourished or at nutritional risk, surgical patients may be at risk of becoming malnourished due to the extensive stress of radical cystectomy and the post-operative response. The question whether immunonutrition is most beneficial for those patients who are malnourished or for those with adequate pre-operative nutritional status remains unanswered. The optimal timing, duration, and immunonutrition supplement composition is unknown. Furthermore, those who undergo neoadjuvant chemotherapy may further present as a subset of patients where immunonutrition may have a larger impact, however given the small study size, such a subset analysis was not possible. Lastly, an important aspect to consider is the cost of immunonutrition and immunonutrition pathways. Unfortunately, most cost-effectiveness analyses have focused on comparing enteral with parenteral feeding. There remains a need to evaluate the cost-effectiveness of various immunonutrition protocols in management of surgical patients.

In a recent review, postoperative immunonutrition in radical cystectomy was not associated with significant benefits outside of early return to a post-operative normal diet (12). Furthermore, since there is a paucity of data evaluating malnourished patients undergoing urological surgery in the nutritional supplement setting, there is no established consensus on the role of immunonutrition. Although the current investigators demonstrated in this small pilot study an impact of SIM on peak IL-6 and plasma arginine levels, the clinical benefit as it pertains to inflammatory-modulated complications and postoperative recovery remains speculative. Until there are 
further randomized trials or well-controlled comparative effective studies evaluating the utility of immunonutrition supplementation, immunonutrition in patients with bladder cancer undergoing radical cystectomy should be considered investigational.

\section{Acknowledgements}

None.

\section{Footnote}

Conflicts of Interest: The authors have no conflicts of interest to declare.

\section{References}

1. Parekh DJ, Reis IM, Castle EP, et al. Robot-assisted radical cystectomy versus open radical cystectomy in patients with bladder cancer (RAZOR): an open-label, randomised, phase 3, non-inferiority trial. Lancet 2018;391:2525-36.

2. Cerantola Y, Valerio M, Hubner M, et al. Are patients at nutritional risk more prone to complications after major urological surgery? J Urol 2013;190:2126-32.

3. Johnson DC, Riggs SB, Nielsen ME, et al. Nutritional predictors of complications following radical cystectomy. World J Urol 2015;33:1129-37.

4. Gregg JR, Cookson MS, Phillips S, et al. Effect of preoperative nutritional deficiency on mortality after radical cystectomy for bladder cancer. J Urol 2011;185:90-6.

5. Skeie E, Koch AM, Harthug S, et al. A positive association

Cite this article as: Bjurlin $M A$, Smith $A B$, Huang WC. Impact of immunonutrition on radical cystectomy immunoresponse and outcomes; opportunity for peri-operative optimization. Transl Androl Urol 2018;7(Suppl 6):S760-S762. doi: 10.21037/tau.2018.08.09 between nutritional risk and the incidence of surgical site infections: A hospital-based register study. PLoS One 2018;13:e0197344.

6. Bertrand J, Siegler N, Murez T, et al. Impact of preoperative immunonutrition on morbidity following cystectomy for bladder cancer: a case-control pilot study. World J Urol 2014;32:233-7.

7. Drover JW, Dhaliwal R, Weitzel L, et al. Perioperative use of arginine-supplemented diets: a systematic review of the evidence. J Am Coll Surg 2011;212:385-99.e1.

8. Hamilton-Reeves JM, Stanley A, Bechtel MD, et al. Perioperative Immunonutrition Modulates Inflammatory Response after Radical Cystectomy: Results of a Pilot Randomized Controlled Clinical Trial. J Urol 2018;200:292-301.

9. Hamilton-Reeves JM, Bechtel MD, Hand LK, et al. Effects of Immunonutrition for Cystectomy on Immune Response and Infection Rates: A Pilot Randomized Controlled Clinical Trial. Eur Urol 2016;69:389-92.

10. Lyon TD, Turner IIR, McBride D, et al. Preoperative immunonutrition prior to radical cystectomy: a pilot study. Can J Urol 2017;24:8895-901.

11. Maffezzini M, Gerbi G, Campodonico F, et al. A multimodal perioperative plan for radical cystectomy and urinary intestinal diversion: effects, limits and complications of early artificial nutrition. J Urol 2006;176:945-8; discussion 948-9.

12. Munbauhal G, Drouin SJ, Mozer P, et al. Malnourishment in bladder cancer and the role of immunonutrition at the time of cystectomy: an overview for urologists. BJU Int 2014;114:177-84. 